\title{
Incidence, Prevention and Management of Post Tonsillectomy Hemorrhage
}

\author{
Ali Abdallah Abdel Rahman, Mohammad Amin Al-Morsy, Mohamed Said Hussein \\ Department of Otolaryngology, Faculty of medicine, Al-Azhar University \\ Corresponding author: Mohamed Said Hussein, email: mohammedsaidhussien63@gmail.com
}

\begin{abstract}
Background: Tonsillectomy is one of the most frequently performed operations in otolaryngology, especially in children. Basically, bleeding within $24 \mathrm{~h}$ has been classified as primary bleeding whereas bleeding occurring from the next day after surgery has been classified as secondary bleeding.

Aim of the Work: Was to review the possibility of decreasing the incidence of post- tonsillectomy hemorrhage along with the associated complications.

Methods: This is a prospective study enrolling one thousand and two hundred patients undergoing tonsillectomy (with or without adenoidectomy in Al- Azhar University Hospitals (El-Hussien - Sayed Jalal - AL Zahraa) from April 2018 till December 2018. We restricted the study to full time participants and resident staff so that complete records would be available.

Results: The mean age of patients was 13.53 years, $47.62 \%$ of patients were male while $52.38 \%$ of patients were female. Primary bleeding occurred in 16 cases while secondary bleeding occurred in 32 cases. There are significant differences among studied groups as regard to control methods of post-tonsillectomy bleeding which could be conservative or operative.

Conclusion: We concluded that patients with post-tonsillectomy bleeding should return for clinical evaluation as they may need conservative management or possible operation interference either for ligation or cautery of bloody tonsil, that trigger a need for external carotid artery ligation.
\end{abstract}

Keywords: Bleeding, Cautery, Tonsillectomy, Sutures

\section{Introduction}

Tonsillectomy is one of the most frequently performed operations in otolaryngology, especially in children ${ }^{(1)}$.

Since bleeding after tonsillectomy may lead to reoperation, the need for transfusion, and even death, it is particularly important to give it special attention. The incidence of bleeding after tonsillectomy is approximately $0.5-10 \%$, with deaths occurring in 1 in 20,000 patients $^{(2)}$.

Primary bleeding is likely caused by inappropriate surgical techniques, insufficient hemostasis and the exposure of small blood vessels ${ }^{(3)}$.

Secondary post-tonsillectomy bleeding (PTB), defined as any bleeding that occurs 24 hours or longer after surgery, is estimated to occur in approximately $2-5 \%$ of patients undergoing tonsillectomy. The incidence increases with age as well as being more common if the indication for surgery was recurrent tonsillitis ${ }^{(4)}$.

Bleeding within $24 \mathrm{~h}$ has been classified as

primary bleeding whereas bleeding occurring from the next day after surgery has been classified as secondary bleeding. The clinical symptoms of primary bleeding are more severe than those of secondary bleeding because of the possible risk of aspiration or laryngospasm and invisible swallowing of blood with a consequent circulatory failure ${ }^{(5)}$.

\section{Aim of the Work}

In this study we aimed to review the possibility of decreasing the incidence of posttonsillectomy hemorrhage along with the associated complications.

\section{Patients and Methods}

This is a prospective study enrolling 1200 patients undergoing tonsillectomy (with or without adenoidectomy) and admitted to AlAzhar University Hospitals (El-Hussien - Sayed Jalal - Al-Zahraa) regardless to their age. Out of which, 48 patients had post-tonsillectomy bleeding. Approval of the ethical committee and a 
written informed consent from all the subjects were obtained.

All patients were subjected to close observation as follows:

- Careful history taking: full demographic data, full otolaryngologic symptoms, full patient's history, and full family history.

- Full otorhinolaryngologic examination.

- Preoperative investigations: Complete Blood Count, Bleeding profile and blood sugar for surgical fitness.

- Operative technique either cold dissection or cauterization method with hemostasis technique by ligation or cauterization.

In cases of post tonsillectomy bleeding (PTB), all patients underwent the following:

- Local assessment: By looking at the tonsillar bed and any surrounding source of bleeding in the oral cavity or in the oropharyngeal tract.

- General assessment: Pulse, temperature, blood pressure, the color of the face, orientation of himself and surroundings. This should be done every 30 minutes in the first 6 hours then every hour in the first one day.

- Urgent investigations: Hemoglobin level and cross matching were done for all cases. Hemoglobin level was repeated daily.

- Blood units were packed for each patient on his admission.

Postoperative: patients were divided into the following: a) Non-bleeding patients

b) Patients with primary hemorrhage that required return to operative room

c) Patients with primary hemorrhage that required conservative management.

d) Patients with secondary hemorrhage that required return to operative room.

e) Patients with secondary hemorrhage that required conservative management.

19 patients were prepared for operation to control the bleeding either by stitching or cautery to the site of bleeding. One case needed for External carotid artery ligation.

\section{Statistical Analysis:}

Data was collected and coded to facilitate data manipulation and double entered into Microsoft Access and data analysis was performed using SPSS software version 18 under windows 7. Simple descriptive analysis in the form of numbers and percentages for qualitative data, and arithmetic means as central tendency measurement, standard deviations as measure of dispersion for quantitative parametric data, and inferential statistic test:

- For quantitative parametric data: Independed student $\mathbf{t}$-Test used to compare measures of two independent groups of quantitative data

- Paired t-test in comparing two dependent quantitative data.

- Bivariate Pearson correlation test to test association between variables

- The level $\boldsymbol{P} \leq \mathbf{0 . 0 5}$ was considered the cut-off value for significance.

\section{Results}

Table (1): Demographic data of PTB

\begin{tabular}{|l|c|c|}
\hline Variables & \multicolumn{2}{|c|}{ Total cases No. = 1200} \\
\hline Age (Years) & \multicolumn{2}{|c|}{$13.53 \pm 9.89$} \\
\cline { 2 - 3 } Mean \pm SD & No & Percentage \\
\cline { 2 - 3 } & & $47.62 \%$ \\
Gender & 572 & $52.38 \%$ \\
Female & 628 & \\
\hline
\end{tabular}

In our research, we found out that the mean age of patients had post-tonsillectomy bleeding was 13.53 years, $47.62 \%$ of patients were male and $52.38 \% 37.5 \%$ of patients were female as shown in table (1). 
Table (2): Timing of PTB

\begin{tabular}{|l|c|c|}
\hline \multirow{2}{*}{ Timing of bleeding } & \multicolumn{2}{|c|}{ Patients with PTB $=48$} \\
\cline { 2 - 3 } & Primary & Secondary \\
\hline First 8 hours & 10 & - \\
\hline 8-24 hours & 6 & - \\
\hline 3-5 days & - & 8 \\
\hline Above 5 days & - & 24 \\
\hline
\end{tabular}

As in our study, we found out that 16 cases presented in the first 24 hours while 32 cases presented above 3 days as shown in table (2).

Table (3): Type of tonsillectomy techniques in cases of PTB

\begin{tabular}{|l|c|c|c|}
\hline \multirow{2}{*}{ Techniques } & \multicolumn{2}{|c|}{$\begin{array}{c}\text { Cases had tonsillectomy = 1200 } \\
\text { No. of PTB cases = 48 }\end{array}$} & \multirow{2}{*}{-value } \\
\cline { 2 - 3 } & Bleeding & Not bleeding & \\
\cline { 2 - 3 } & No. & No. & \\
\hline $\begin{array}{l}\text { Technique of tonsillectomy } \\
\text { dissection }\end{array}$ & & & \\
Cold dissection & 732 & 23 & \multirow{2}{*}{$0.028 S$} \\
Electrocautery & 420 & 25 & \\
\hline
\end{tabular}

S: significant

In our study, 755 cases had tonsillectomy with cold dissection 23 cases only had PTB while 445 cases had tonsillectomy with bipolar cauterization, 25 cases only had PTB as shown in table (3).

Table (4): Type of tonsillectomy dissection in cases of PTB

\begin{tabular}{|l|c|c|c|}
\hline \multirow{2}{*}{} & \multicolumn{2}{|c|}{ No. of PTB cases $=48$} & \multirow{2}{*}{$\begin{array}{c}\text { p- value } \\
\text { (Chi-square test) }\end{array}$} \\
\cline { 2 - 3 } & Primary & Secondary & \\
\hline Cold dissection & 13 & 10 & $0.001 \mathrm{~S}$ \\
\hline Electrocautery & 3 & 22 & \\
\hline
\end{tabular}

S: significant

In our study, 13 cases had primary PTB with cold dissection technique while 10 cases had secondary PTB with cold dissection technique. 3 cases only had primary PTB with electrocautery technique while 22 cases had secondary PTB with electrocautery technique, as shown in table (4).

Table (5): Follow up of PTB cases

\begin{tabular}{|l|c|c|c|}
\hline \multirow{2}{*}{$\begin{array}{l}\text { Control of bleeding in cases of } \\
\text { PTB }\end{array}$} & \multicolumn{2}{|c|}{ No. of PTB cases = 48 } & \multirow{2}{*}{ p- value } \\
\cline { 2 - 3 } & Primary & Secondary & \\
\hline Conservative & 4 & 25 & $0.013 \mathrm{~S}$ \\
\hline $\begin{array}{l}\text { Return to OR } \\
\text { (for hemostasis by ligation or } \\
\text { electrocautery) }\end{array}$ & 12 & 5 & \\
\hline Need for ECA ligation & 0 & 2 & \\
\hline
\end{tabular}

\section{S: significant}

In our study, 29 cases had just conservative hospital care while 19 cases had returned to operating room to control bleeding with diathermy or ligation. Two of these patients had secondary hemorrhage to be re- 
operated for ligation of external carotid artery as shown in table (5).

Table (6): Need for blood transfusion

\begin{tabular}{|l|l|l|l|}
\hline \multirow{2}{*}{$\begin{array}{l}\text { Blood transfusion in } \\
\text { cases of PTB }\end{array}$} & \multicolumn{2}{|l|}{ No. of PTB cases $=48$} & p-value \\
\cline { 2 - 3 } & Primary & Secondary & \\
\hline Given & 3 & 5 & 0.784 \\
\hline Not given & 13 & 27 & \\
\hline
\end{tabular}

In this study 3 cases only with primary PTB had blood transfusion, while 16 cases were hospitalized for primary PTB, however 5 cases with secondary PTB had blood transfusion. Overall, 32 cases were hospitalized for secondary PTB as shown in table (6).

\section{Discussion:}

Provided that tonsillectomy is of high frequency, it's indispensable that any surgeon who intends to practice it must have the due knowledge of the possible complications may find.

In the present study, the mean age of patients who had post-tonsillectomy bleeding was 13.53 years, $47.6 \%$ of patients were male and $52.38 \%$ of patients were female.

Likewise, Tomkinson et al. ${ }^{(6)}$ reported that in patients over 12 years of age, the risk of postoperative bleeding (primary or secondary), that required a surgical treatment, was 1,5 to 3 times greater than the population of patients under 12 years of age.

As in our study, we found out that 16 cases presented in the first 24 hours while 32 cases presented above 3 days as shown in table (2).

The literature reported by Silva et al. ${ }^{(7)}$ an incidence of hemorrhage in the immediate postoperative to delayed PTM of $0.28 \%$ to $7.48 \%$, which confirms our study assuming that 11 cases represent an incidence of $1.32 \%$ of hemorrhage in the first 24 hours as from the surgery.

Raut et al. ${ }^{(8)}$, found out that $5.55 \%$ of cases had primary hemorrhage and $16.76 \%$ had secondary hemorrhage that depends on that the only method was cold dissection and hot homeostasis. In other side, $3.14 \%$ of cases had primary hemorrhage and $12.5 \%$ had secondary hemorrhage that depends on that the only method was hot dissection and cold homeostasis ${ }^{8}$.

Our results showed significant differences between cold dissection and electrocautery techniques used for tonsillectomy according to occurrence of PTB and type of this PTB either primary or secondary as shown in tables $(3,4)$. In our study, 29 cases had just conservative hospital care while 19 cases had returned to operating room to control bleeding with diathermy or ligation. Two of these patients had secondary hemorrhage to be re-operated for ligation of external carotid artery as shown in table (5).

All patients, except for those who needed surgical review due to immediate postoperative hemorrhage, remained interned for about 8 hours, with routine prescription of analgesic and antibiotics and if necessary, antiemetic agents, aiming at the reduction of pain, fever and vomits and promoting a quicker return to the usual diet.

Although in a recent study by Burton et al. (9), after a large literature review, concluded that there is no evidence that the use of antibiotics reduces pain or hemorrhages after tonsillectomy operations. In those who evolved with bleeding, the patients remained 24 hours in hospital under suitable observation and reposition of fluids and electrolytes ${ }^{9}$.

Negm et al. ${ }^{(10)}$, about $90 \%$ of group B cases needed conservative management without intervention. Only four (10\%) cases needed to reenter the operation theater, and ligation of the bleeder under general anesthesia was performed.

In this study 3 cases only with primary PTB had blood transfusion and additionally 5 cases with secondary PTB had blood transfusion as shown in table (6). Mutz and Simon ${ }^{(11)}$ found out that only one $(0.12 \%)$ case required a unit of red blood cells concentrate for the adequate 
hemodynamic balance, which is within the average in the literature studies, this states the number of the cases that needed blood transfusion ranged from $0 \%$ to $2.3 \%$.

\section{Conclusion:}

The exact incidence of PTB is very difficult to determine as statistics in literature range from $0 \%-20 \%$ while in the present study the incidence was $4 \%$. Sex, method of tonsillectomy dissection or hemostasis, indication for the operation and use of postoperative antibiotics were not significantly associated with risk of PTB while patients older than 12 years significantly suffered from increased risk of BTP.

Also, we concluded in our study that patients with post-tonsillectomy bleeding should return for clinical evaluation as they may need conservative management or possible operation interference either for ligation or cautery of bloody cases, that could call for external carotid artery ligation.

\section{References}

1. Katharine K, Phil E, Pablo P et al. (2012): Effect of tranexamic acid on surgical bleeding: systematic review and cumulative meta-analysis. BMJ., 344:30-54.

2. Krishna S, Hughes LF and Lin SY (2003): Postoperative hemorrhage with nonsteroidal anti-inflammatory drug use after tonsillectomy: a metaanalysis. Arch. Otolaryngol. Head Neck Surg., 129:10861089.

3. Lee MS, Montague ML and Hussain SS (2004): Post-tonsillectomy hemorrhage: cold versus hot dissection. Otolaryngol. Head Neck Surg., 131:833-6.
4. Arora R, Saraiya S, Niu X et al. (2015): Post tonsillectomy haemorrhage: Who needs intervention? Int. J. Pediatr. Otorhinolaryngol., 79 (2):165-9

5. Randall DA, Hoffer ME (1998): Complications of Tonsillectomy and Adenoidectomy. Otolaryngol. Head Neck Surg., 118(1):61-8.

6. Tomkinson A, Harrison W, Owens D et al. (2011): Risk factors for postoperative hemorrhage following tonsillectomy. Laryngoscope, 121(2):279-88.

7. Silva BS, Garcia LB, Ortiz LR et al. (2009): Hemorrhage in the Adenoidectomy and/or Tonsillectomy Immediate Postoperative Intl. Arch. Otorhinolaryngol., (13)9:155-160.

8. Raut VV, Bhat N, Sinnathuray AR et al. (2002): Bipolar scissors versus cold dissection for pediatric tonsillectomy - a prospective, randomized pilot study. Int. J. Pediatr. Otorhinolaryngol., 64: 9-15.

9. Burton MJ, Archer SM and Rosenfeld RM (2008): Extracts from the cochrane library: antibiotics to reduce posttonsillectomy morbity? Arch. Ololaryngol. Head and Neck Surgery, 139(1):7-9.

10. Negm H, Atef A, Lasheen $H$ et al. (2017): Factors affecting secondary posttonsillectomy hemorrhage: a case-control study. Egypt J. Otolaryngol., 33:50-5.

11. Mutz I and Simon H (1993): Hemorrhage complications of the tonsillectomy and adenoidectomy. Experiences with 7.743 operation in 14 years. Wien. Klin. Wochenschr., 105:520-2. 